\title{
WAVEFORM COMPARISON AND NONLINEARITY SENSITIVITIES OF FBMC, UFMC AND W-OFDM SYSTEMS
}

\author{
Changyoung An, Byeongjae Kim, and Heung-Gyoon Ryu \\ Department of Electronics Engineering, Chungbuk National University, \\ Cheongju, Korea 361-763 \\ acy890217@naver.com, bj5236@nate.com, and ecomm@cbu.ac.kr
}

\begin{abstract}
Recently, new waveforms for the 5th generation cellular system have been studied in many ways. UFMC, FBMC (filter bank multi-carrier) and W-OFDM (window orthogonal frequency division multiplexing) waveforms are very strong candidates as a new waveform for $5 G$ system. In this paper, we have evaluated the spectrum characteristic and BER performance of the waveforms under the effect of nonlinear HPA. Also, we like to show the comparison of the timefrequency resources of each system because it would be very important to estimate the spectral efficiency and communication throughput. As simulation results, it is confirmed that $O O B$ power of each system increases, and $O O B$ power increase of FBMC system is the biggest. Additionally, we have confirmed that performance of every system is degraded by strength of HPA nonlinearity, and every system needs the PAPR reduction method for the nonlinear distortion compensation and power saving, even though it would be more complicated. Comparison table for the time-frequency resources requirement for the each modulation systems is included.
\end{abstract}

\section{KEYWORDS}

new waveform; OFDM; FBMC; UFMC; HPA nonlinearity

\section{INTRODUCTION}

The mobile traffic is being increased dramatically, because various mobile devices and multimedia services are being increased [1].Also, the growth of mobile traffic is being accelerated. It is difficult for the present mobile communication to support the mobile traffic required in the future [2].In order to solve the problem, studies for next generation 5G mobile communication has been carried actively [3-4].

Conventional orthogonal frequency division multiplexing (OFDM) based on multi-carrier has high-power out-of-band (OOB) [5]. This characteristic causes adjacent channel interference (ACI). OFDM uses a wide guard band in order to avoid ACI. It decreases spectral efficiency when a number of mobile devices simultaneously access a base station. Next generation mobile communication system requires high-level key performance indicators (KPIs). It is difficult for OFDM to satisfy the KPIs. Universal filtered multi-carrier (UFMC) and filter bank based multicarrier (FBMC) are known as the candidate waveform for 5G mobile communication. When the

Natarajan Meghanathan et al. (Eds) : NETCOM, NCS, WiMoNe, GRAPH-HOC, SPM, CSEIT - 2016 pp. 83- 90, 2016. (C) CS \& IT-CSCP 2016

DOI : $10.5121 /$ csit.2016.61508 
f-OFDM suggested by Huawei appeared in the first place, the filtered-OFDM system adopted one-filter system for the sharper OOB (out-of-band) spectrum characteristics, but they changed into the multiple filter system, which became very similar to the UFMC(universal filtered multicarrier) system. These systems use filtering technique based on multi-carrier. These techniques have characteristic of low OOB power in comparison with conventional OFDM. Therefore, these systems have high spectrum efficiency. FBMC uses a filtering technique in each sub-carrier. UFMC uses a filtering technique in each sub-band [8-9].

However, these systems based on OFDM are vulnerable to non-linearity of high-power amplifier (HPA), like OFDM. OFDM has high peak-to-average power ratio (PAPR) because multi-carrier signals are overlapped. High PAPR causes nonlinear distortion in HPA because it saturates HPA. Similarly, UFMC and FBMC have high PAPR because these systems are based on multi-carrier [10-11]. In UFMC and FBMC system, if nonlinear distortion is caused by high PAPR, OOB power of these systems is increased. That is, advantage of these systems vanishes. Therefore, this drawback should be overcome in the candidate techniques for $5 \mathrm{G}$ mobile communication.

In this paper, in order to overcome the drawback, we focus on spectrum characteristic analysis and performance evaluation of FBMC and UFMC system under the effect of nonlinear HPA. Firstly, we describe and explain OFDM, UFMC, FBMC system. And then, we design the systems. Next, under linear environment, we analyse the spectrum characteristic of each system and evaluate bit error rate (BER) performance of each system. And then, under the effect of nonlinear HPA, we analyse spectrum characteristic of each system and evaluate bit error rate (BER) performance of each system.

Also, we like to show the comparison of the time-frequency resources of each system because it would be very important to estimate the spectral efficiency and communication throughput.

\section{SYSTEM MODEL}

\subsection{OFDM}

In OFDM system, firstly, in transmitter of OFDM system, the data symbols are transformed into parallel stream from series stream by S/P block. The changed symbols are mapped onto each subcarrier by inverse fast Fourier transform (IFFT) operation. After IFFT operation, the timedomain signals are transformed into series stream from parallel stream by P/S block. And then, cyclic prefix (CP) is added in order to reduce the effect of inter-symbol interference (ISI). Finally, the RF signals are amplified by high-power amplifier (HPA). Receiver of OFDM system consists of reversed structure in comparison with OFDM transmitter. Additionally, in OFDM receiver, an equalizer is used in order to restore desired signal. The equalizer is very simple because of CP. OFDM receiver uses one-tap equalizer. OFDM system requires simple equalizer with one tap [12]. However, each subcarrier of OFDM system has high side-lobe power. As a result, channel capacity is decreased in OFDM system [12].

\subsection{UFMC}

UFMC filters each sub-band that consists of orthogonal multi-carrier in order to reduce OOB power [6]. In the UFMC system, each sub-band signal is transformed into series stream by P/S. Secondly, in UFMC receiver, the received signal is applied to RF chain. The received signal is transformed into baseband signal by RF chain. Baseband signal is converted into digital signal by ADC. And then, time-domain pre-processing is processed. After the process, the series data stream is transformed into a parallel data stream by S/P. The time-domain parallel data stream is converted to frequency-domain stream by $2 \mathrm{~N}$-FFT operation [6]. After 2N-FFT operation, odd- 
numbered data symbols are selected and equalized. Spectrum of UFMC system has lower OOB power in comparison with spectrum of OFDM system. This is good advantage. However, because UFMC system uses multi-carriers and multi-carriers are overlapped, UFMC system has high PAPR. High PAPR characteristic can distort signal of UFMC system [6].

\subsection{FBMC}

FBMC system filters each sub-carrier in order to reduce OOB power of spectrum [7]. In FBMC system, firstly, in transmitter of FBMC system, data symbols are transformed into parallel stream from series stream by S/P. The parallel symbols are modulated to offset quadrature amplitude modulation (OQAM) signal [7]. The modulated OQAM signal is transformed into a signal filtered by each sub-carrier by using the synthesis filter bank that consists of IFFT and poly phase network (PPN) [7]. Finally, the amplified FBMC signal is transmitted by antenna. Receiver of FBMC system consists of reversed structure in comparison with FBMC transmitter. FBMC system has lower OOB power in comparison with UFMC system and OFDM system. This is a good advantage. However, FBMC system has high system complexity. Additionally, because FBMC system uses multi-carrier, it has high PAPR.

\subsection{W-OFDM}

W-OFDM is a improved version of OFDM system. In the W-OFDM system, it does not use the filter but it uses the extension and windowing method on each OFDM symbol in order to reduce OOB power of spectrum.

\subsection{HPA nonlinearity}

In this paper, purposes are spectrum characteristic analysis and performance evaluation of OFDM, UFMC, FBMC and W-OFDM system under the effect of nonlinear HPA. Therefore, we have designed each system. In Saleh model, characteristics of AM-AM and AM-PM are as follows [13].

$$
\begin{gathered}
G[A(t)]=\frac{\alpha_{A} A(t)}{1+\beta_{A} A(t)^{2}} \\
\Phi[A(t)]=\frac{\alpha_{\phi} A(t)^{2}}{1+\beta_{\phi} A(t)^{2}}
\end{gathered}
$$

Equation (1) shows AM-AM characteristic of Saleh model, nonlinear HPA model. A is amplitude

of input signal. $\alpha_{A}$ and $\beta_{A}$ are coefficients for adjusting amplitude of output signal. Equation (2) shows AM-PM characteristic of Saleh model. ${ }^{\alpha}{ }_{\dot{\phi}}$ and ${ }^{\beta}{ }_{\dot{\phi}}$ are coefficients for adjusting phase of output signal.

The following formatting rules must be followed strictly. This (.doc) document may be used as a template for papers prepared using Microsoft Word. Papers not conforming to these requirements may not be published in the conference proceedings.

\section{SIMULATION RESULTS}

Table 1 shows simulation parameters. 
Table 1. Simulation parameters.

\begin{tabular}{|l||l|}
\hline Parameter & Value \\
\hline Modulation & QPSK \\
\hline \# of total subcarrier & 64 \\
\hline \# of used subcarrier & 32 \\
\hline \# of null subcarrier & 32 \\
\hline & Phydyas prototype \\
Filter for FBMC & $\mathrm{H} 0=1$ \\
& $\mathrm{H} 1=0.97196$ \\
& $\mathrm{H} 2=0.7071$ \\
\hline \multirow{2}{*}{ Filter for UFMC } & $\mathrm{H} 3=0.235147$ \\
\hline \# of sub-band in UFMC & Chebyshev \\
\hline \# of used sub-band in UFMC & Attenuation $=60 \mathrm{~dB}$, Length $=10$ \\
\hline
\end{tabular}

Table 2 shows the considered HPA nonlinear conditions. Condition 0 is linear. Conditions 1 to 5 are nonlinear condition. Condition 1 is weak nonlinear condition. Condition 5 is strong nonlinear condition.

Table 2. Condition of HPA nonlinearity.

\begin{tabular}{|l|l|l|}
\hline Condition & AM-AM & AM-PM \\
\hline \multirow{2}{*}{$\begin{array}{l}\text { (Linear) } \\
\text { Nonlinear1 }\end{array}$} & $\alpha_{i=1}$ & $\alpha_{\infty}=0$ \\
\cline { 2 - 3 } & $\beta_{i=0}$ & $\beta_{\infty}=0$ \\
\cline { 2 - 3 } & $\beta_{i=0.04}$ & $\alpha_{\infty}=0.26$ \\
\hline \multirow{2}{*}{ Nonlinear2 } & $\alpha_{i=1}$ & $\beta_{\infty}=15.9$ \\
\cline { 2 - 3 } & $\beta_{i=0.2}$ & $\alpha_{\infty}=0.26$ \\
\hline \multirow{2}{*}{ Nonlinear3 } & $\alpha_{i=1}$ & $\beta_{\infty=2.38}$ \\
\cline { 2 - 3 } & $\beta_{i=0.4}$ & $\alpha_{\infty}=0.26$ \\
\hline \multirow{2}{*}{ Nonlinear4 } & $\alpha_{i=1}$ & $\beta_{\infty}=0.69$ \\
\cline { 2 - 3 } & $\beta_{i=0.6}$ & $\alpha_{\infty}=0.26$ \\
\hline \multirow{2}{*}{ Nonlinear5 } & $\alpha_{i=1}$ & $\beta_{\infty=0.127}$ \\
\cline { 2 - 3 } & $\beta_{i=0.8}$ & $\alpha_{\infty}=0.26$ \\
\hline
\end{tabular}

Table 3. Comparison of OOB power characteristic.

\begin{tabular}{l|l|l|l|l}
\hline Condition & OFDM & UFMC & FBMC & W-OFDM \\
\hline \hline Linear & $-26 \mathrm{~dB}$ & $-83 \mathrm{~dB}$ & $-120 \mathrm{~dB}$ & $-90 \mathrm{~dB}$ \\
\hline condition 1 & $-26 \mathrm{~dB}$ & $-82 \mathrm{~dB}$ & $-85 \mathrm{~dB}$ & $-85 \mathrm{~dB}$ \\
\hline condition 2 & $-26 \mathrm{~dB}$ & $-74 \mathrm{~dB}$ & $-75 \mathrm{~dB}$ & $-75 \mathrm{~dB}$ \\
\hline condition 3 & $-26 \mathrm{~dB}$ & $-66 \mathrm{~dB}$ & $-67 \mathrm{~dB}$ & $-69 \mathrm{~dB}$ \\
\hline condition 4 & $-26 \mathrm{~dB}$ & $-61 \mathrm{~dB}$ & $-62 \mathrm{~dB}$ & $-63 \mathrm{~dB}$ \\
\hline
\end{tabular}

Table 3 shows OOB power comparison about each system. In this table, we have confirmed as follows. Under the HPA nonlinearity environment FBMC system shows the biggest change of OOB power, and OFDM system shows the smallest change of OOB power. 
Figs. 1 to 4 show BER performances of each system. Each system has ideal performance under the linear condition or nonlinear condition. Under the nonlinear HPA environment, BER performance of every system is degraded. Additionally, FBMC system shows the smallest degradation of BER performance. However, even though FBMC system is the strongest against HPA nonlinearity, every system needs the PAPR reduction method for the nonlinear distortion compensation and power saving.

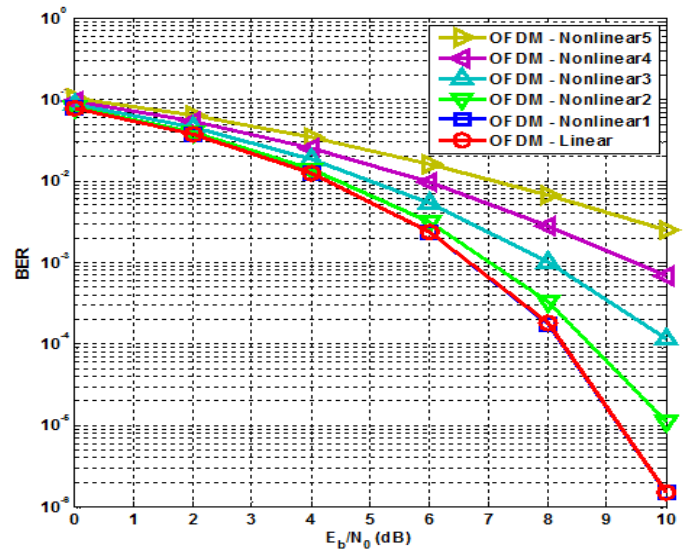

Figure 1. BER of OFDM system according in HPA conditions.

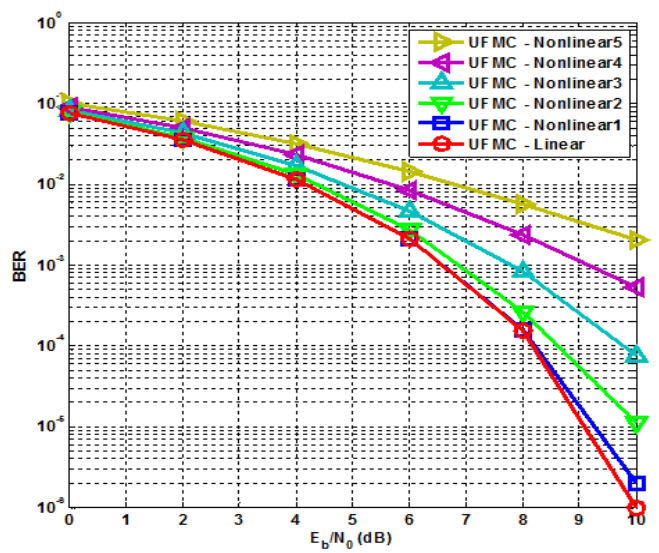

Figure 2. BER of UFMC system according in HPA conditions.

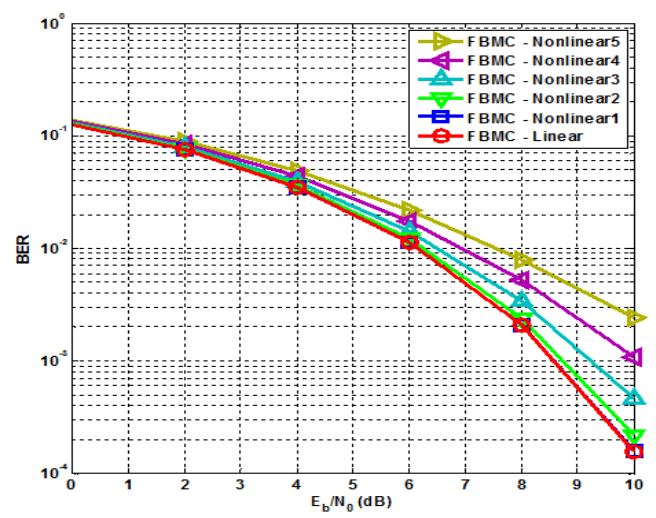

Figure 3. BER of FBMC system according in HPA conditions. 


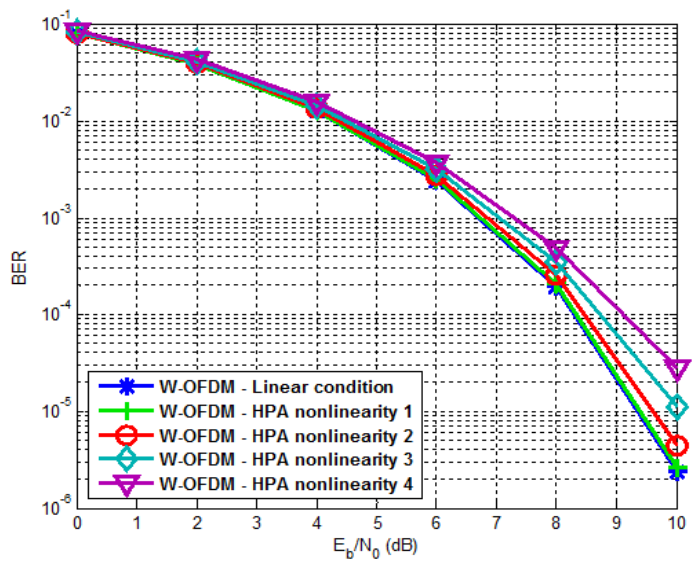

Figure 4. BER of W-OFDM system in HPA conditions.

Next, in order to compare the time-frequency resources of each candidates system, we have set some necessary conditions as in the below. Also, in the Table 4, we provide the comparison for the time-frequency resources requirement for the each modulation systems.

Allocated bandwidth $=20 \mathrm{MHz}$

\# of used sub-carriers $=16$

\# of transmission bits $=128$

4QAM modulation(2bit) $* 16$ sub-carrier $* 4$ synthesis symbols $=128$ bits

iFFT size $=64, \mathrm{CP}$ length $=9$

FBMC, Overlapping Factor $(\mathrm{K})=4$

$\mathrm{W}-\mathrm{OFDM}$, Extension length $=6$

OOB emission suppression (Frequency, 7.5Mhz Offset) / TTI length (Time)

Table 4. Comparison of the time-frequency resources.

\begin{tabular}{|c|c|c|c|c|}
\hline & OFDM & UFMC & FBMC & W-OFDM \\
\hline Linear & $-26 \mathrm{dBc} / 292$ & $-83 \mathrm{dBc} / 292$ & $-130 \mathrm{dBc} / 480$ & $-66 \mathrm{dBc} / 304$ \\
\hline HPA condition 1 & $-26 \mathrm{dBc} / 292$ & $-82 \mathrm{dBc} / 292$ & $-85 \mathrm{dBc} / 480$ & $-66 \mathrm{dBc} / 304$ \\
\hline HPA condition 2 & $-26 \mathrm{dBc} / 292$ & $-74 \mathrm{dBc} / 292$ & $-75 \mathrm{dBc} / 480$ & $-66 \mathrm{dBc} / 304$ \\
\hline HPA condition 3 & $-26 \mathrm{dBc} / 292$ & $-66 \mathrm{dBc} / 292$ & $-67 \mathrm{dBc} / 480$ & $-65 \mathrm{dBc} / 304$ \\
\hline HPA condition 4 & $-26 \mathrm{dBc} / 292$ & $-63 \mathrm{dBc} / 292$ & $-65 \mathrm{dBc} / 480$ & $-63 \mathrm{dBc} / 304$ \\
\hline
\end{tabular}

\section{CONCLUSIONS}

FBMC and UFMC systems are the strong modulation candidate for $5 \mathrm{G}$ mobile communication system. Since these systems are basically multicarrier system, it is important to study the nonlinearity sensitivity. In this paper, we have focused on spectrum characteristic analysis and BER performance evaluation of OFDM, FBMC, and UFMC system under the effect of nonlinear HPA. As simulation results, we have confirmed that if HPA nonlinearity rises in each system, OOB power of each system increases. The OOB power increase of FBMC system is the biggest. Additionally, we have confirmed that performance of every system is degraded by strength of HPA nonlinearity, and every system needs the PAPR reduction method for the nonlinear distortion compensation and power saving, even though it would be more complicated. Also, we like to show the comparison of the time-frequency resources of each system because it would be 
very important to estimate the spectral efficiency and communication throughput. We provide the comparison table for the time-frequency resources requirement for the each modulation systems.

\section{ACKNOWLEDGEMENTS}

"This research was supported by Basic Science Research Program through the National Research Foundation of Korea (NRF) funded by the Ministry of Education, Science and Technology(No.2013R1A2A2A01005849) and This work was supported by Institute for Information \& communications Technology Promotion (IITP) grant funded by the Korea government(MSIP) (No. R0101-16-244, Development of 5G Mobile Communication Technologies for Hyper-connected Smart Services)"

\section{REFERENCES}

[1] Shanzhi Chen; Jian Zhao, "The requirements, challenges, and technologies for 5G of terrestrial mobile telecommunication," Communications Magazine, IEEE, vol. 52, no. 5, pp. 36-43, May 2014.

[2] Dahlman, E.; Mildh, G.; Parkvall, S.; Peisa, J.; Sachs, J.; Selén, Y.; Sköld, J., "5G wireless access: requirements and realization," Communications Magazine, IEEE, vol. 52, no. 12, pp. 42-47, December 2014

[3] G. Wunder et al., "5GNOW: non-orthogonal, asynchronous waveforms for future mobile applications”, IEEE Commun. Mag., vol. 52, no. 2, pp. 97-105, Feb. 2014.

[4] P. Banelli et al., "Modulation Formats and Waveforms for the Physical Layer of 5G Wireless Networks: Who Will be the Heir of OFDM?", in arXiv:1407.5947, July 2014.

[5] Schaich, F.; Wild, T., "Waveform contenders for 5G - OFDM vs. FBMC vs. UFMC," Communications, Control and Signal Processing (ISCCSP), 2014 6th International Symposium on, pp. 457-460, 21-23 May 2014.

[6] Vakilian, V.; Wild, T.; Schaich, F.; ten Brink, S.; Frigon, J.-F., "Universal-filtered multi-carrier technique for wireless systems beyond LTE," in Globecom Workshops (GC Wkshps), 2013 IEEE, pp. 223-228, 9-13 Dec. 2013.

[7] Farhang-Boroujeny, B., "OFDM Versus Filter Bank Multicarrier," in Signal Processing Magazine, IEEE, vol. 28, no. 3, pp. 92-112, May 2011.

[8] Wonsuk Chung; Beomju Kim; Moonchang Choi; Hyungju Nam; Hyunkyu Yu; Sooyoung Choi; Daesik Hong, "Synchronization Error in QAM-Based FBMC System," in Military Communications Conference (MILCOM), 2014 IEEE, pp. 699-705, 6-8 Oct. 2014.

[9] Mukherjee, Mithun; Shu, Lei; Kumar, Vikas; Kumar, Prashant; Matam, Rakesh, "Reduced out-ofband radiation-based filter optimization for UFMC systems in 5G," in Wireless Communications and Mobile Computing Conference (IWCMC), 2015 International, pp. 1150-1155, 24-28 Aug. 2015.

[10] Kollar, Zs.; Varga, L.; Czimer, K., "Clipping-Based Iterative PAPR-Reduction Techniques for FBMC," in OFDM 2012, 17th International OFDM Workshop 2012 (InOWo'12); Proceedings of , pp. 1-7, 29-30 Aug. 2012.

[11] Chafii, M.; Palicot, J.; Gribonval, R., "Closed-form approximations of the PAPR distribution for Multi-Carrier Modulation systems," in Signal Processing Conference (EUSIPCO), 2014 Proceedings of the 22nd European, pp. 1920-1924, 1-5 Sept. 2014. 
[12] Elshirkasi, A.M.; Siddiqi, M.U.; Habaebi, M.H., "Generalized Discrete Fourier Transform Based Minimization of PAPR in OFDM Systems," in Computer and Communication Engineering (ICCCE), 2014 International Conference on, pp. 205-208, 23-25 Sept. 2014.

[13] P. Drotar, J. Gazda, D. Kocur, and P. Galajda, "MC-CDMA performance analysis for different spreading codes at HPA Saleh model,” 18th Int. Conf. Radioelektronika, pp. 1-4, Prague, Apr. 2008.

\section{AUTHORS}

Changyoung An was born in Chungbuk, Republic of Korea in 1989. He received the B.S. degree and M.S. degree in the department of electronic engineering from Chungbuk National University in February 2013 and February 2013, respectively. He is currently working toward the Ph.D degree at the department of Electronic Engineering, Chungbuk National University, Republic of Korea. His research interests include wireless communication system, signal processing, antenna technology and 5G mobile communication.

Byeongjae Kim was born in Gyeongbuk, Republic of Korea in 1992. He received the B.S. degree in the department of electronic engineering from Chungbuk National University in 2016. Now he is currently working toward the master's degree at the department of Electronic Engineering, Chungbuk National University, Republic of Korea. His research interests include wireless communication system, signal processing.

Heung-Gyoon Ryu (M'88) was born in Seoul, Republic of Korea in 1959. He received the B.S. and M.S. and Ph.D. degrees in electronic engineering from Seoul National University in 1982, 1984 and 1989. Since 1988, he has been with Chungbuk National University, Korea, where he is currently Professor of Department of Electrical, Electronic and Computer Engineering in Chungbuk National University. And he worked as chief director of RICIC (research institute of computer, information communication center) in Chungbuk National University from March 2002 to Feb 2004. His main research interests are digital communication systems, communication circuit design,
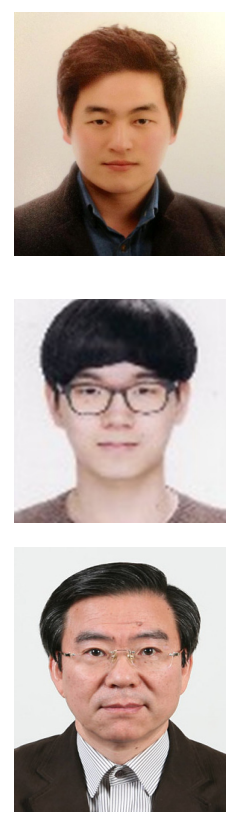
spread spectrum system and communication signal processing. Since 1999, he has worked as reviewer of the IEEE transaction paper. He received the '2002 ACADEMY AWARD' from the Korea Electromagnetic Engineering Society, Korea. He received the "BEST PAPER AWARD" at the 4th International Conference on Wireless Mobile Communications (ICWMC 2008) Athens, Greece, July 27-Aug.1, 2008. Also, He received the "BEST PAPER AWARD" at the International Conference on Advances in Satellite and Space Communications (SPACOMM 2009), Colmar France, July 20-25, 2009. 